\title{
A Mobile Rehabilitation Application for the Remote Monitoring of Cardiac Patients after a Heart Attack or a Coronary Bypass Surgery
}

\author{
Valérie Gay \\ Faculty of Engineering and IT, \\ University of Technology Sydney \\ PO box 123, Broadway \\ 2007 NSW Australia \\ +61296144645 \\ Valerie.Gay@uts.edu.au
}

\author{
Peter Leijdekkers Faculty of \\ Engineering and IT, University of \\ Technology Sydney PO box 123, \\ Broadway \\ 2007 NSW Australia \\ +61296147871
}

Peter.Leijdekkers@uts.edu.au

\author{
Edward Barin Cardiology \\ Department, Royal North Shore \\ Hospital, Sydney \\ Westbourne Av St Leonards NSW \\ 2065 Australia \\ +61294113930
}

Edward.barin@gmail.com

\begin{abstract}
This paper describes a personalised rehabilitation application using a smart phone (PDA) and wireless (bio) sensors. It instructs and motivates patients to follow their exercise programme and keeps track of their progress. It also monitors the relevant biosignals and provides immediate feedback to the patient.

Sensors transmit data to the mobile phone where it is analysed locally and the data can also be instantaneously transmitted to a healthcare centre for remote monitoring by a health professional.

The rehabilitation application is personalised for each cardiac patient and provides tailored advice (e.g. exercise more, slow down). A trial with a rehabilitation centre is in progress in which we investigate whether the personalised rehabilitation application improves the success of the rehabilitation programme in terms of patient compliance with recommended life style changes (such as increase physical activity or lose weight) and whether use of the system brings peace of mind to cardiac patients.
\end{abstract}

\section{Categories and Subject Descriptors}

J.3 [Computer Applications]: Life and Medical sciences - Health

\section{General Terms}

Measurement, Design, Experimentation, Human Factors.

\section{Keywords}

Cardiac rehabilitation, Remote monitoring.

\section{INTRODUCTION}

Cardiac rehabilitation programmes aim at maximizing physical, psychological and social functioning of a patient following a myocardial infarction or coronary bypass surgery [1].

Typically, a cardiac rehabilitation programme includes counselling to help the patient achieve lifestyle changes (give up smoking, lose weight, change diet, learn to reduce stress, cope with depression, anger and stress during recovery). It provides support and training to help the patient to return to normal work and activities and it teaches patients how to manage their personal heart condition. It also includes supervised and monitored gym sessions to improve the patient's cardiovascular fitness. The exercises are tailored to the patient based on medical evaluation and, for the longer term, a personalised home exercise programme is given to the patient. Heart rate and blood pressure need to be monitored and traditional rehabilitation programmes take a baseline measurement of health at the start of the programme, with follow-up measurements at regular intervals [2].

The benefits of rehabilitation have been demonstrated by several research groups [3, 4, 5]. It can reduce mortality, lower blood pressure, improve lipids and stress levels [6]. However, despite strong evidence that cardiac rehabilitation reduces disability and prolongs life, fewer than one in five people in the USA receive rehabilitation services after a heart attack or coronary bypass surgery [7]. This is sometimes due to access issues (geography, lack of resources or ill-health) but often due to lack of motivation since it is hard to make lifestyle changes without any external help. Another issue is that many patients do not complete the rehabilitation programme and pull out before the desired health style changes have been achieved.

For people having access issues, home based cardiac rehabilitation has been widely noted as a viable alternative to rehabilitation programmes conducted at a hospital [8]. A study [3] in Canada and Australia found that remote monitoring could help patients that do not benefit from in-house rehabilitation services. It also showed that the use of remote monitoring of physiological data helps reduce heart failure admissions to hospitals and lowers the mortality rate by nearly twenty per cent for chronic heart failure patients. Their study highlighted that remote monitoring 
has the potential to significantly improve clinical outcomes (mortality, morbidity and quality indicators).

To improve long term clinical outcomes, patients need to make lifestyle changes and this is not something that can be achieved in a few weeks. The patients need to integrate exercises in their daily life; they need to understand the cause and effect of medication and activities on their health. It is important for the patients to keep track of their health and be reminded and motivated to exercise.

Several commercial products enable patients to keep track of their physical activities using a watch, mobile phone or iPod. For example, the iPod/Nike pedometer [9] tracks the user using an accelerometer sensor on a shoe with a wireless connection to an iPod. The information collected can be transferred to the user's PC. Mobile phones such as NTT DoCoMo Fujitsu Pedometer Phone [10] or the Nokia 5500 Sports [11] integrate Personal Trainer type of applications in the mobile phone. Products such as Polar Heart Rate Monitors [12] and GARMIN Forerunner 50 [13] are used by joggers to keep track of the heart rate and provide basic feedback to the user (e.g., heart rate, calorific output distance, speed). While such products can provide some useful functions such as monitoring the cardiac patient's activity, they are aimed at the sports market and as such are not tailored for cardiac patients specifically; hence it is not surprising that they do not provide the full range of functionality needed for remote cardiac monitoring by a health professional.

Internet based applications such as Microsoft HealthVault [14] or Google Health [15] allow individuals to enter their bio data manually and keep track of their activity and progress. This could be used by health professionals to assess the progress made by a patient but it involves a lot of discipline and time commitment from patients to enter their data manually after each exercise or measurement.

Substantial research has been done on mobile monitoring systems, which typically incorporate wearable or implanted sensors and run software on a mobile device to perform a set of functions around one clinical application. CardioMobile [16] defines a system for real-time remote monitoring of ECG, heart rate, location and speed. The CSIRO e-Health project [8] focuses on the assessment of cardiac rehabilitation patients in a community care model. The main differences between our mobile rehabilitation application and existing ones are the following: our application combines activity and biosignal monitoring (e.g. exercise, ECG, weight, blood pressure, glucose). It provides immediate local feedback to the patient without the intervention of a health professional. It can also be used for real-time remote monitoring of a patient using 3G technology. Health professionals can use a standard web browser to access their patients' data and carry out remote monitoring and reporting. Our rehabilitation application is integrated into a platform that offers useful functionalities such as arrhythmia detection and emergency facilities such as automatic emergency calls in case of detection of a fall or of a life-threatening arrhythmia.

This paper presents our mobile rehabilitation application for remote monitoring of cardiac patients after a heart attack or a coronary bypass surgery. It is organised as follows; Section 2 describes the Personal Health Monitor application and highlights the characteristics that make it suitable for cardiac rehabilitation,
Section 3 details the rehabilitation application and presents the user interface. Section 4 concludes this paper.

\section{The Personal Health Monitor}

The Personal Health Monitor (PHM) provides personalised, intelligent, non-intrusive, real time health monitoring using wireless sensors and a mobile phone (see Figure 1). The application and its algorithms have been developed by our team at the University of Technology, Sydney $[17,18,19]$.

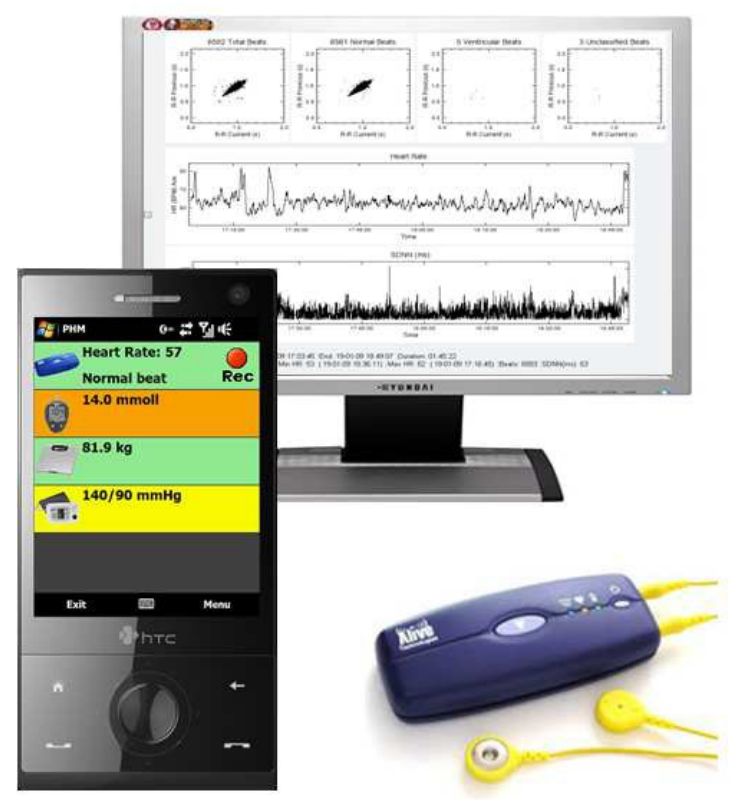

Figure 1. Personal Health Monitor System

To date, over 70 low to medium risk cardiac patients, aged 22 to 90 years old, have used the Personal Health Monitor in a trial with the Sydney Royal North Shore Hospital (Cardiology Department) for Cardiac Rhythm Monitoring. In this trial, patients are given a heart monitor and mobile phone to monitor and record their cardiac rhythms for several weeks in their normal environment (see Figure 2).

The PHM offers the following functionalities which makes it attractive for remote cardiac rehabilitation:

Ambulatory monitoring: Using small sensors and a phone it allows convenient, non intrusive monitoring for a prolonged period of time, while users carry on with their normal daily routines. The trial showed that most patients have no difficulty using a mobile phone and ECG sensor and they found the application straightforward to use. Those patients leading an active life appreciated the fact that no one could see they were being monitored and wearing sensors. Ambulatory monitoring is useful for patients who are not close to a rehabilitation centre and would otherwise miss out in joining a rehabilitation group. Additionally, rehabilitation programmes typically run for six weeks and it is important to keep up the exercises after the rehabilitation programme finishes.

Multiple sensors: Many different sensors are supported. The wireless sensors can be either attached to the user's body (e.g. ECG and Accelerometer) or can be external devices (e.g. Blood 
Pressure Monitor or Weight Scale), that are used when required. Users can use all, or a selected group, depending on their health condition and personal preferences. The following sensors are available: ECG, Fall detector, Pulse Oximeter, Blood Pressure, Weight and Blood Glucose. The PHM uses off-the-shelf sensors. They are available on the market and their technology is mature. The user needs a Microsoft Windows mobile phone, and may buy or rent the sensors. The user can download the software onto the mobile phone and use it just like any other windows mobile application. Another advantage of off-the-shelf sensors is that health professionals trust these devices since they are FDA, TGA and/or CE, approved. The patient can claim the sensor rental or purchase from Medicare or private health insurance in some countries (e.g. in Australia, the cost of a blood pressure monitor and blood glucose monitor can be claimed on health insurance).

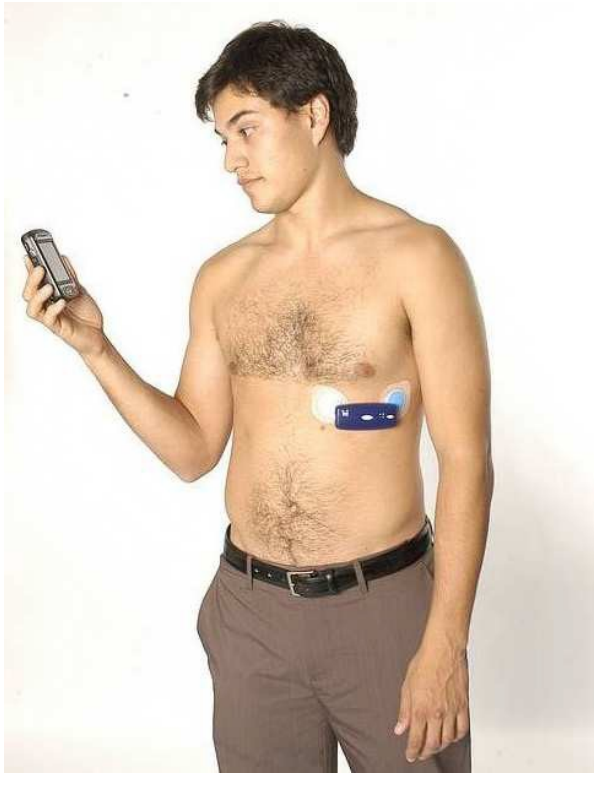

Figure 2. Patient with PHM phone and ECG sensor

Personalisation: Each user has different needs and preferences and the PHM application can be configured to the patients' and health professionals' needs and requirements.

Instant feedback: The Personal Health Monitor analyses and stores biosignals and activity data on the phone and provides instant personal feedback to the user. If required, the phone can be set, in the event of a cardiac arrest, to loudly play a message with pre-set CPR instructions for any bystander, so that they know how to assist. The trial highlighted that the type of feedback not only depends on the clinical application but also on the patient's profile. Some patients want to be in charge of their health, they are aware of medical terminology and wish to get immediate feedback. Others prefer to use the application with a minimum of interaction because they find it stressful. During the trial we discovered that elderly patients living alone preferred to have audio reminders and warnings, whereas more socially active patients preferred the application to be as unobtrusive as possible ('silent mode'). The flexibility of the PHM user interface allows personalisation of feedback to the user.
Software running locally on the phone: The Personal Health Monitor software runs on the user's mobile phone and analyses the data received from the sensors in real time. For the younger generation, using a mobile phone for local processing of the sensor data is a natural choice. For the elderly, it could be expected that simpler devices would be more suitable. However, during the trial, elderly patients were able to use the application even if they had never used a mobile phone before. Simplicity and motivation seem to be the key prerequisites for patient acceptance of the technology, not age. The patients had no real issues with the battery life of the mobile phone. Most people nowadays are used to recharging their phone regularly.

Arrhythmia detection: The PHM application can detect and record various arrhythmias and can react to serious arrhythmias such as ventricular fibrillation/tachycardia. The trial has demonstrated that the detection of important cardiac arrhythmias is feasible using the PHM system when compared to conventional Holter monitors. (24h loop, no feedback). The ECG signal quality is in the majority of cases of sufficient quality for a cardiologist to make an assessment.

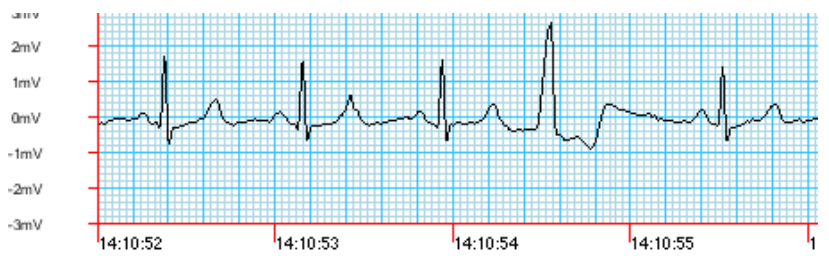

Figure 3. ECG trace from PHM system

Reminders and logs: The Personal Health Monitor software allows the users to set reminders for their measurements and to keep logs of their activities. The trial showed that patients who used the PHM to monitor their wellbeing found it useful to be in charge of their health and keep track of their progress (e.g. they can work out what triggers changes in their blood pressure). This is confirmed by a study [20] which showed that people who check their own blood pressure tend to be more conscious of the importance of taking their medication on a regular basis. They tend to notice what type of food or activities raise their blood pressure and they will adjust their lifestyle to avoid them, leading to better results.

Communication: The sensor data and analysis can be instantaneously sent to the Health Care data server using 3G or any other Internet connection available on the phone. The PHM application can also be configured to automatically ring or SMS pre-assigned numbers in emergency situations, such as when it detects a life threatening arrhythmia or a fall. The PHM application can use either wireless or wired communication for synchronising data.

Remote Monitoring via Health Care data server: Specialists can remotely monitor their patients via the Health Care data server. Data sent from the phone with details about the user's condition can be used for diagnosis. Specialists can remotely adjust the parameters and tailor them to each patient's situation. Health specialists and patients can use secure web access to access the data. 
Reliability: The trial showed that reliability and ease of use are paramount in order for an application to be accepted by patients and health professionals. Feedback received from the trial so far suggests that we achieved that goal.

\section{PHM - REHABILITATION APPLICATION}

This section describes a typical scenario for a person having experienced a heart attack and the enrolment into a rehabilitation programme. It outlines how the PHM rehabilitation application can assist in recovering from the cardiac event.

\subsection{Scenario}

We conduct this research in cooperation with two cardiac rehabilitation centres in Sydney (Australia). The following scenario shows how our rehabilitation application could be integrated in their current outpatient and maintenance programmes.

Jack has recently experienced an acute cardiovascular event and joins a rehabilitation programme to exercise under supervision and receive information on how to make lifestyle changes. On entering the programme he is assessed on several factors such as

Physical and psychosocial condition

Symptom recognition

Cardiac risk factors

Past medical history

He performs a six minute walk where his Rate of Perceived Exertion (RPE) is determined as well as his O2 saturation and heart rate. The results form the basis of his exercise programme. Jack is advised to be more active, to lose weight and to monitor his diabetes and hypertension.

Jack is also given information about the mobile rehabilitation application. Since he leads an active life and lives far from the centre he chooses to use the mobile rehabilitation application since he can then be monitored more than twice a week. He already owns a 3G mobile phone and the physiotherapist advices him to rent a heart monitor, a blood pressure monitor, a blood glucose monitor and a weight scale.

A technician installs the remote rehabilitation software on his phone and pairs the Bluetooth sensors with his phone. He also personalises Jacks application based on Jack's profile and personal requirements (e.g. max heart rate for the exercises, health condition). This can be done on the phone or using the web interface.

Jack attends a 20 minute tutorial together with the other patients who opted for the remote monitoring service. He gets instructed on how to use the mobile application and sensors and how to synchronise his data with the health centre server. A four page quick guide with screenshots is handed out that summarizes the main information.

Twice a week, Jack discusses his recovery progress with a nurse and a physiotherapist assists him with his cardiovascular and muscle strengthening exercises. Jack wears the heart monitor and uses the mobile monitoring application during the exercises. This allows him to become familiar with the application while he is still being supervised. It also allows the physiotherapist to adjust threshold parameters for the exercises.

After a couple of weeks, Jack has gained enough confidence to do the exercises at his local gym using the mobile rehabilitation application. He synchronizes the data every week with the health centre server. He still attends the information sessions about dietetics, medication, stress management and cardiac risk factors at the centre.

At the end of the six weeks programme, Jack is assessed on the progress made and correct use of the mobile rehabilitation application. Jack is encouraged to make use of community programmes and to continue exercising.

Six months later, Jack returns for a reassessment. The centre has access to all his data and can easily assess his progress. If there are concerns the GP or cardiologist are contacted. A report is routinely prepared for Jack's GP.

\subsection{User Interface}

The PHM user interface consists of three different parts: live data, configuration and rehabilitation. Navigation and operation of the application is touch based. Figure 4 shows the live data screen. Here users can monitor their current heart rate and beat classification as well as other physiological signals such as blood pressure, weight and blood glucose.

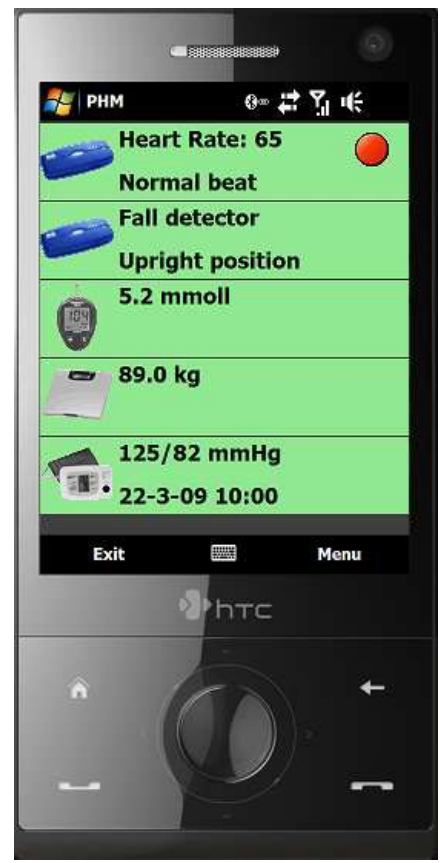

Figure 4. Live data screen

Sensors can be activated or deactivated based on the patient's condition. In day to day use of the PHM this is the only screen used by patients. Other information such as reminders and battery status of sensors are displayed here as well. The user can be warned by audio if issues arise with the sensors or measurements are outside a predefined range. 
The Administration interface enables the patient or the specialist to personalise the application. Here sensors can be configured and (de)activated and user preferences can be changed. The PHM application supports remote management of the application via the website. This enables a specialist to update threshold settings remotely, therefore the patient does not need to come in with the hardware for adjustments (see Figure 5).
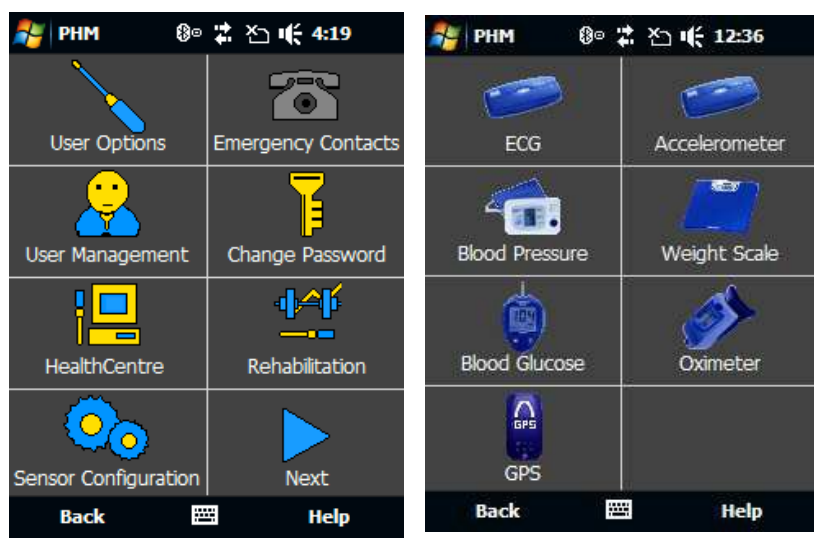

Figure 5. Configuration of PHM

The Rehabilitation section allows the activation and configuration of the exercises. Figure 6 shows the exercises that are currently available. The selection of exercises is based on discussions with the Royal North Shore Hospital Rehabilitation centre. Other exercises can be easily added if required. It also shows how a particular exercise can be tailored for a patient. The specialist can set the duration, the target heart rate that should be achieved but also the maximum heart rate which would signal the user to slow down or stop the exercise. Other parameters such as target speed, grade etc can be set to instruct the patient on how to configure the exercise equipment. Reminders can be configured to remind the user when it is expected to perform the exercise.
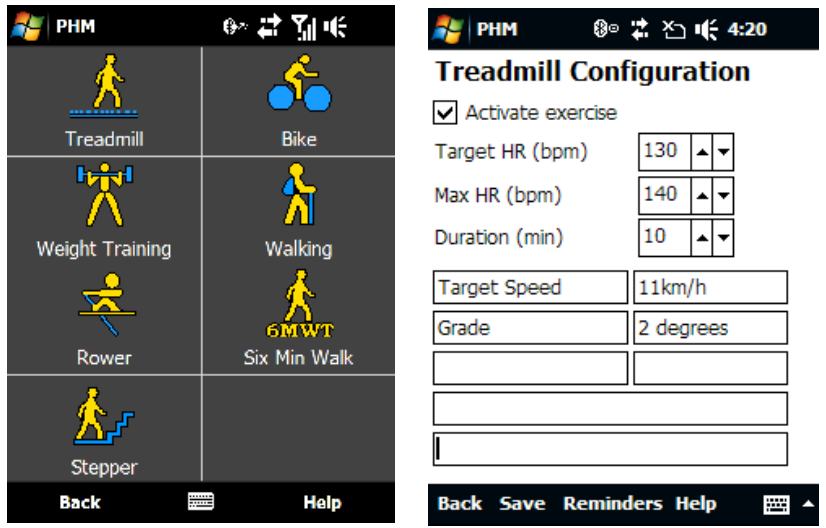

Figure 6. Configuration of Rehabilitation Exercises

The patient can perform the exercises with or without the heart monitor. If the heart monitor is used, the heart rate is monitored and the user knows when to push or slow down. The patient is also monitored for arrhythmia abnormalities and an ECG will be recorded if an abnormality is detected.
The user selects the required exercise and taps START when ready (see Figure 7). The application guides the user through the exercise. It keeps track of the thresholds set for the particular exercise. It motivates the patient to speed up or slow down or stay within the recommended thresholds. It also acts as a stopwatch.

\begin{tabular}{|c|c|}
\hline Q7 PHM & $\Leftrightarrow Y_{1}$ 㕰 \\
\hline Tread & \\
\hline Grade & 5 \\
\hline Calories & 350 \\
\hline $\begin{array}{l}\text { Time } \\
\text { 00:00:00 }\end{array}$ & $\begin{array}{l}\text { Target } \\
\text { 00:30:00 }\end{array}$ \\
\hline Heart Rate & Target \\
\hline 68 & 145 \\
\hline $\begin{array}{l}\text { Press Start } \\
\text { when ready }\end{array}$ & Start \\
\hline
\end{tabular}

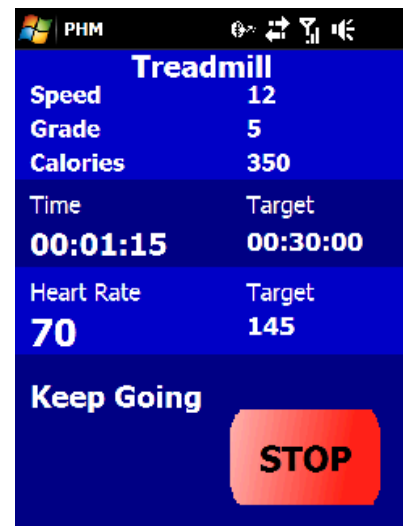

Figure 7. Performing an exercise

After completing the exercise the application will ask several questions to assess the perceived difficulty. The Borg Rating of Perceived Exertion (RPE) scale is used which is a useful adjunct to the heart rate as an intensity guide for cardiac exercise training. RPE is a subjective rating that the user assigns to the intensity of his/her exercise based on their perception of how hard the physical exertion was (see Figure 8).

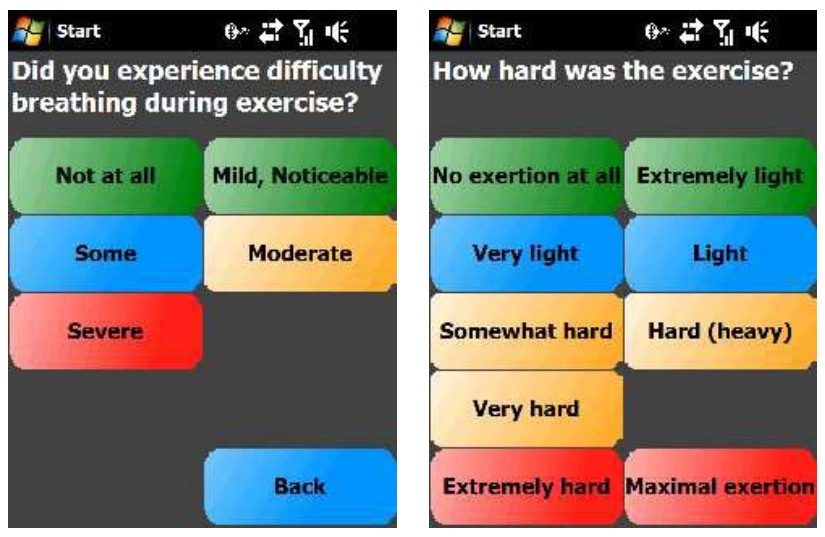

Figure 8. Rating of Perceived Exertion

We also capture symptoms such as chest pain, nausea, dizziness, confusion, shortness of breath, blurred vision since they can be of significance to the specialist. 


\subsection{Remote Monitoring}

The PHM application can be used standalone on the PDA where the user keeps track of their progress. If the patient participates in a cardiac rehabilitation programme the data can also be uploaded to a web site (www.PersonalHeartMonitor.com) where health professionals can remotely monitor the patient. The data is transferred to the web site and potential new thresholds and exercise information set by the Rehabilitation Health centre are transferred to the phone. Synchronisation between the mobile phone and the web site is done at predefined intervals or instantly using 3G.

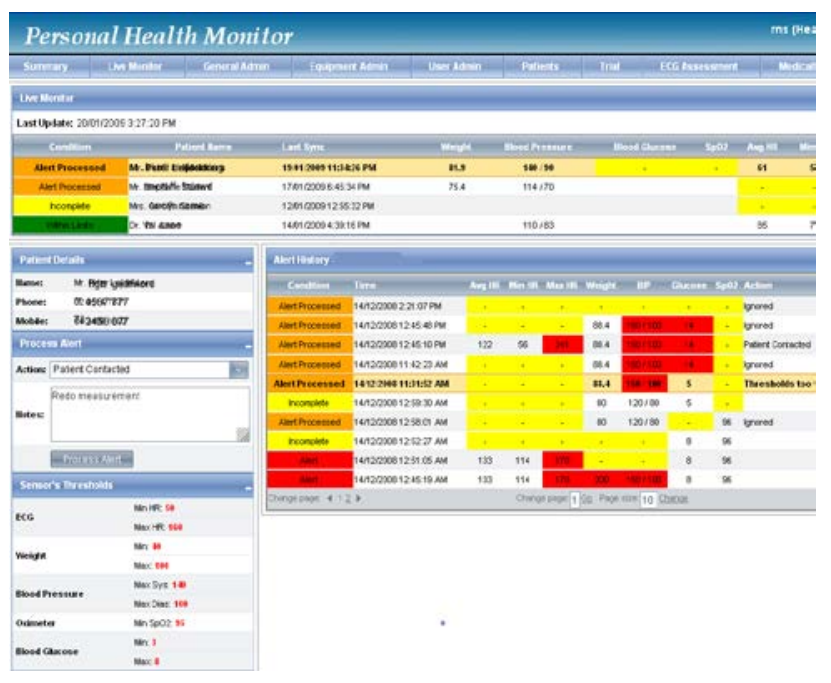

Figure 9. 24/7 Remote monitoring via PHM website

Access to the web site is secure and accessible by patients and authorised health professionals. Patients can view their data in graphical form and view the comments/diagnosis made by the health professional. Qualified staff can monitor 24/7 the physiological data from patients and are alerted if measurements are over the thresholds as set for the patient. Patients needing their attention appear on top of the list (see Figure 9).

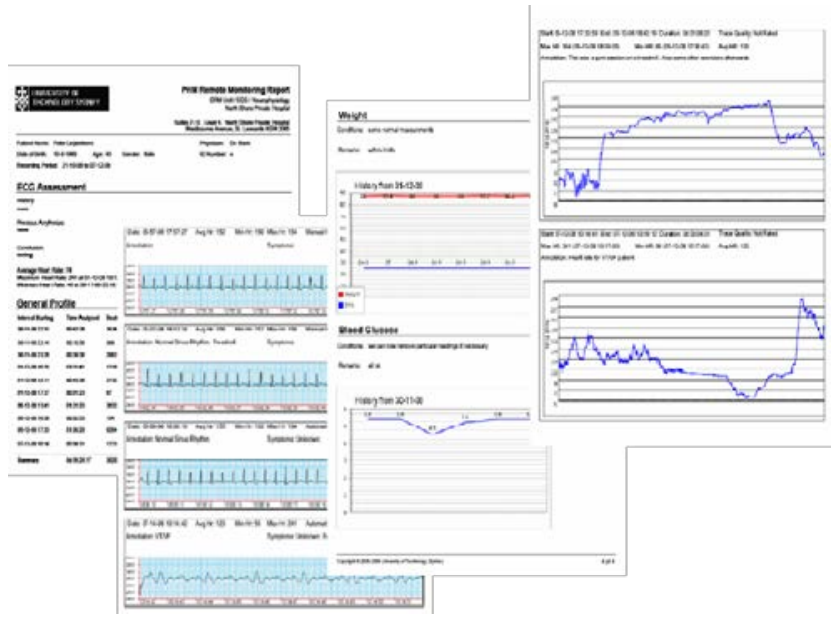

Figure 10. Reports
The exercise information is also transferred to the website so that a specialist can review progress but also to adjust exercise parameters and add comments. Keeping track of the exercise history will show the patients' progress over time and hopefully motivate a patient to continue with the exercises.

Rehabilitation staff can view and comment on the physiological data of their patients and reports can be generated for review by other specialists (see Figure 10).

\section{CONCLUSIONS}

The mobile rehabilitation application described in this paper aims at encouraging patients to do their exercises and improve their confidence by constant local monitoring. It can aid patients to achieve the required life style changes by monitoring their process but also to remind them to do their exercises.

We are conducting trials with the Royal North Shore Hospital in Sydney, Australia and two associated rehabilitation clinics. The trial focuses on the usability and acceptance of mobile phones and wearable sensors by patients. We also receive feedback on the usability of the website by health professionals and improvements are made to satisfy their requirements and needs.

Future research focuses on automatic detection of physical activity levels of the patient and to make the mobile application as pervasive as possible. We will also adapt the user interface based on the trial feedback and new functionalities will be added to assist patients setting and reviewing their personal weekly goals. Future improvements include the introduction of music as a feedback mechanism similar to MPT train [21] and the creation of a virtual network that would allow people from the same group to stay in touch and discuss their goals and achievements with their peers.

Our application can be used by rehabilitation clinics (in-house) but also by outpatients that have not access to rehabilitation services or wish to continue after the in-house sessions. It does not replace rehabilitation clinics since human supervision and personal health checks are essential to the rehabilitation process. It complements those services by recording the patient's biosignals and activities and provides data to rehabilitation centres to remotely evaluate the patient's health and progress. We believe that this application can help cardiac patients making long term lifestyle changes.

\section{ACKNOWLEDGMENTS}

The Personal Health Monitor is supported by the University of Technology, Sydney and is currently under commercial development. The authors would in particular thank Michael Ascharsobi for his contribution to the cardiac rehabilitation application and Val Jones for reviewing this script. 


\section{REFERENCES}

[1] American Heart Association. Cardiac Rehabilitation. http://www.americanheart.org/presenter.jhtml?identifier=304 7638

[2] National Heart foundation of Australia and Australian Cardiac Rehabilitation Association. (2004). Recommended framework for cardiac rehabilitation.

[3] University of Alberta. "Remote Monitoring Saves Lives Of Heart Patients, Research Shows."ScienceDaily 23 April 2007.

[4] Thompson, D. R., Bowman, G. S., Kitson, A.L., Bono, D. P. de, Hopkins, A. (1996). Cardiac rehabilitation in the United Kingdom: guidelines and audit standards. Heart. Vol. 75. pp. 89-93

[5] Horgan, J., Bethel, H., Carson, P., Davidson, C., Julian, D., Mayou, R. A., Bagle, R. (1992). Working party report on cardiac rehabilitation. British Heart Journal. Vol 67. pp. 412418

[6] Thomson, D.R., Bono, D. P. (1999). How valuable is cardiac rehabilitation and who should get it? Heart. Vol 82. pp. 545-546.

[7] Normand S-L. T., Ades P., and Prottas J., Rehabilitation significantly underused after heart attack and bypass surgery, Circulation: Journal of the American Heart Association, 25Sep-2007.

[8] Bidargaddi N.P, Sarela A., Activity and heart rate-based measures for outpatient cardiac rehabilitation, Methods of Information in Medicine 2008; 47(3):208-216.
[9] Nike iPod Sport Kit, http://www.apple.com/ipod/nike.

[10] NTT pedometer phone http://www.nttdocomo.com/pr/2007/001323.html.

[11] Nokia. www.nokia.com .

[12] Polar heart rate monitoring equipment, http://www.polar.fi .

[13] Garmin. www.garmin.com .

[14] Healthvault. Healthvault.com .

[15] Google health. www.google.com/health .

[16] Cardiomobile - Remote Monitoring System, June 2008, http://www.ihbi.qut.com/research/injury/cardiomobile/index.

[17] Gay V., Leijdekkers P., 'A Health Monitoring System Using Smart Phones and Wearable Sensors', Special Issue on 'Smart Sensors in Smart Homes' International Journal of Assistive Robotics and Mechatronics, Vol. 8, No. 2, 2007.

[18] Fokkenrood S., Leijdekkers P., Gay V., 'Ventricular Tachycardia/Fibrillation Detection Algorithm for 24/7 Personal Heart Monitoring', ICOST 2007, June, 2007 Nara, Japan.

[19] Personal Health Monitor http://www.personalhealthmonitor.net

[20] Michael A.Weber, Hypertension Medicine, published Totowa, N.J. : Humana, c2001. ISBN 0896037886.

[21] Oliver N., Flores-Mangas F., MPTrain: a mobile, music and physiology-based personal trainer, Proceedings of the 8th conference on Human-computer interaction with mobile devices and services, September 12-15, 2006, Helsinki, Finland 